\title{
Unified Framework of Approximating and Interpolatory Subdivision Schemes for Construction of Class of Binary Subdivision Schemes
}

\author{
Pakeeza Ashraf, ${ }^{1}$ Ghulam Mustafa $\mathbb{D}^{2},{ }^{2}$ Abdul Ghaffar $\mathbb{D D}^{3,4}$ Rida Zahra, ${ }^{2}$ \\ Kottakkaran Sooppy Nisar $\left(\mathbb{1}^{5}\right.$ Emad E. Mahmoud, ${ }^{6,7}$ and Wedad R. Alharbi ${ }^{8}$ \\ ${ }^{1}$ Department of Mathematics, Government Sadiq College Women University, Bahawalpur 63100, Pakistan \\ ${ }^{2}$ Department of Mathematics, The Islamia University of Bahawalpur, Bahawalpur 63100, Pakistan \\ ${ }^{3}$ Informetrics Research Group, Ton Duc Thang University, Ho Chi Minh City, Vietnam \\ ${ }^{4}$ Faculty of Mathematics \& Statistics, Ton Duc Thang University, Ho Chi Minh City, Vietnam \\ ${ }^{5}$ Department of Mathematics, College of Arts and Sciences, Prince Sattam bin Abdulaziz University, Wadi Aldawaser, \\ 11991, Saudi Arabia \\ ${ }^{6}$ Department of Mathematics and Statistics, College of Science, Taif University, P.O. Box 11099 Taif 21944, Saudi Arabia \\ ${ }^{7}$ Department of Mathematics, Faculty of Science, Sohag University, Sohag 82524, Egypt \\ ${ }^{8}$ Physics Department, Faculty of Science, University of Jeddah, Jeddah, Saudi Arabia
}

Correspondence should be addressed to Kottakkaran Sooppy Nisar; ksnisar1@gmail.com

Received 3 November 2020; Revised 25 November 2020; Accepted 1 December 2020; Published 17 December 2020

Academic Editor: Ali Aral

Copyright ( 2020 Pakeeza Ashraf et al. This is an open access article distributed under the Creative Commons Attribution License, which permits unrestricted use, distribution, and reproduction in any medium, provided the original work is properly cited.

\begin{abstract}
In this paper, a generalized algorithm to develop a class of approximating binary subdivision schemes is presented. The proposed algorithm is based on three-point approximating binary and four-point interpolating binary subdivision schemes. It contains a parameter which classifies members of the new class of subdivision schemes. A set of efficient properties, for instance, polynomial generation and reproduction, support, continuity, and Hölder continuity, is discussed. Moreover, applications of the proposed subdivision schemes are given in order to demonstrate their variety, flexibility, and visual performance.
\end{abstract}

\section{Introduction}

Subdivision is a competent way of producing smooth curves or surfaces in geometric modeling and computer graphics. It repeatedly refines the initial polygonal shape. After each split average step, we get closer to the limit curve, which is the limit of an infinite series. A nice property of subdivision schemes is that they are simple and local, which means that local change in initial data will only have a local effect in the resulting object. Subdivision schemes have become celebrated because of their simplicity and efficiency. There are generally two main categories of subdivision schemes: interpolatory and approximating. For interpolating subdivision schemes, limit curve always passes through initial control points while for approximating subdivision schemes it may or may not. Subdivision schemes play an integral role in computer graphics due to their wide range of applications in many fields such as engineering, medical science, space science, graphic visualization, and image processing. Differential equations are used for mathematical modeling of many phenomena. Different techniques are being used to solve boundary value problems [1] and nonlinear problems [2]. Nowadays, subdivision schemes are also becoming a popular tool to numerically solve boundary value problems [3]. Subdivision algorithms are also a major field in many multiscale techniques applied in data compression. In some applications, the given data need not be reproduced at each step of the subdivision process, which needs the applications of subdivision schemes. Several researchers in the area of continuous geometry have been established classical subdivision 
schemes for various kinds of initial control data. In geometric modeling and engineering, practical applications of subdivision curves are restricted due to their shortcomings, and to overcome these shortcomings, a lot of work has been carried out [4-17].

Deslauriers and Dubuc [18] presented a family of interpolating binary subdivision schemes. They used Lagrange interpolating polynomial for construction of schemes. Horman and Sabin (HS) [19] proposed a family of binary subdivision schemes having cubic precision. Some members of the $H S$ family are interpolating and some are approximating. Mustafa et al. [20] offered a family of binary subdivision schemes which has alternating primal and dual symbols. Ashraf et al. [21] discussed a family of binary subdivision schemes based on Lane-Riesenfeld algorithm. Members of the proposed family have quintic precision. Mustafa and Bari [22] developed a family of univariate subdivision schemes for curve generation and data fitting. Asghar and Mustafa [23] presented a unified framework of stationary and nonstationary subdivision schemes. Keeping in view this practice, we present a generalized algorithm to develop a new class of approximating binary subdivision schemes. Ghaffar et al. [24-29] constructed geometric continuity conditions for the construction of free-form generalized subdivision curves with single shape parameter. These free-form complex shape adjustable generalized curves can be obtained by using shape-adjustable generalized subdivision schemes. These newly proposed approaches not only take over the benefits of classical subdivision curve and surface schemes but also resolve the issue of shape adjustability of subdivision curves and surfaces with the help of tension control shape parameters. They modeled some complex curves and surfaces using higher continuity conditions. The proposed masks of the schemes provide an alternative approach to generate the complex curves using higher continuity conditions with simple and straightforward calculation for the proposed algorithm because they are blended with linear polynomials rather than trigonometric functions. In 2020, Ashraf et al. $[17,30,31]$ proposed a new approach using the generalized hybrid subdivision curve with shape parameters to solve the problem in construction of some symmetric curves and surfaces. These curves are easily modified by the changing the values of shape parameters.

In this paper, we offer a Lane-Riesenfeld-like algorithm to derive a class of binary approximating subdivision schemes. Our algorithm is based on the well-known fourpoint interpolating binary subdivision scheme [18], which is $C^{1}$ continuous, and three-point approximating binary subdivision scheme [32], which has $C^{3}$ continuity. Considering $\phi$ smoothing stages as in the Lane-Riesenfeld algorithm, our proposed algorithm allows us to derive a class of univariate subdivision schemes. In fact, each member of the proposed class is enumerated by $\phi$, and higher values of $\phi$ give schemes with wider masks and support, higher continuity, higher Holder regularity, and higher degree of polynomial generation. The first member of the proposed class (corresponding to $\phi=0$ ) coincides with the three-point approximating binary subdivision scheme [32]. The proposed class of schemes generates schemes of higher continuities and visually more smooth limit curves as compared to existing families of schemes. The content of the paper is structured as follows. In Section 2, fundamental definitions and concepts are given. Section 3 presents a generalized algorithm for construction of new class of subdivision schemes. Section 4 is devoted for properties of proposed schemes, such as continuity, Hölder continuity, and support of basic limit function. Geometrical analysis and some beautiful examples of limit curve are given in Section 5. Section 6 presents a summary of the paper.

\section{Preliminaries}

Let the initial data be given by a set of control points $\mathbf{G}^{0}=\left\{g_{i}^{0} \in \mathbb{R}, i \in \mathbb{Z}\right\}$, and the set of control points at refinement level $h(h \geq 0, h \in \mathbb{N})$ is given by $\mathbf{G}^{h}=\left\{g_{i}^{h} \in \mathbb{R}\right.$, $i \in \mathbb{Z}\}$. Define $\mathbf{G}^{h+1}=\left\{g_{i}^{h+1} \in \mathbb{R}, i \in \mathbb{Z}\right\}$ recursively by the following binary refinement rules:

$$
g_{i}^{h+1}=\sum_{k \in \mathbb{Z}} b_{i-2 k} g_{i}^{h}, \quad i \in \mathbb{Z},
$$

where the finite set $\mathbf{B}=\left\{b_{i}, i \in \mathbb{Z}\right\}$ is called mask. The recursive algorithm associated with the repeated application of (1) is called subdivision scheme and denoted by $S$. The Laurent polynomial or symbol of the scheme $S$ is defined as

$$
B(z)=\sum_{l \in \mathbb{Z}} b_{l} z^{l}
$$

Theorem 1 (see [33]). If a binary scheme $S$ is convergent, then the mask $\mathbf{B}=\left\{b_{i}, i \in \mathbb{Z}\right\}$ satisfies

$$
\sum_{l \in \mathbb{Z}} b_{2 l}=\sum_{l \in \mathbb{Z}} b_{2 l+1}=1
$$

The symbol of a convergent scheme can be also be written as

$$
B(z)=B_{\text {even }}\left(z^{2}\right)+z B_{\text {odd }}\left(z^{2}\right),
$$

with $B_{\text {even }}(z)=\sum_{l \in \mathbb{Z}} b_{2 l} z^{l}$ and $B_{\text {odd }}(z)=\sum_{l \in \mathbb{Z}} b_{2 l+1} z^{l}$.

Theorem 2 (see [33]). A binary scheme S associated with the symbol

$$
B(z)=\frac{(z+1)^{m+1}}{2^{m}} L(z)
$$

is said to be $C^{m}$ continuous if the subdivision scheme associated with the symbol $L(z)$ is contractive.

Proposition 3 (see [34]). A binary scheme S generates polynomials of degree $m$ if and only if

$$
B(1)=2, B(-1)=0 \text { and } B^{(j)}(-1)=0, j=1,2, \cdots, m .
$$


TABLE 1: Mask of the $R_{\varphi}$ schemes corresponding to different values of parameter $\varphi$.

\begin{tabular}{lcc}
\hline$\varphi$ & Scheme & Mask \\
\hline 0 & 3-point & $\frac{1}{16}[1,5,10,10,5,1]$ \\
1 & 5-point & $\frac{1}{256}[-1,4,44,124,170,124,44,4,-1]$ \\
2 & 6-point & $\frac{1}{4096}[1,-13,-17,309,1338,2478,2478,1338,309,-17,-13,1]$ \\
3 & 8-point & $\frac{1}{65536}[-1,22,-91,-580,1303,12362,31557,41928,31557,12362,1303,-580,-91,22,-1]$ \\
\hline
\end{tabular}

Proposition 4 (see [34]). A binary scheme S reproduces polynomials of degree $n$ with respect to parametrization $\left\{e_{j}^{h}=(j+\tau) / 2^{h}\right\}_{j \in \mathbb{Z}}$ with $\tau=B^{(j)}(1) / 2$, if and only if it generates polynomials of degree $n$ and

$$
B^{(j)}(1)=2 \prod_{i=0}^{j-1}(\tau-i), \quad j=1,2, \cdots, n .
$$

\section{Algorithm for Construction of Schemes}

The well-known four-point interpolating binary subdivision scheme [18] is given by

$$
\left\{\begin{array}{l}
g_{2 i}^{h+1}=g_{i}^{h}, \\
g_{2 i+1}^{h+1}=-\frac{1}{16} g_{i-1}^{h}+\frac{9}{16} g_{i}^{h}+\frac{9}{16} g_{i+1}^{h}-\frac{1}{16} g_{i+2}^{h}
\end{array}\right.
$$

By considering (4), the symbol of the even part of scheme (8) is as follows:

$$
P_{\text {even }}(z)=\left(\frac{z+1}{2}\right)\left(\frac{-z^{2}+10 z-1}{8}\right)
$$

Now, consider the three-point approximating binary subdivision scheme [32]

$$
\left\{\begin{array}{l}
g_{2 i}^{h+1}=\frac{1}{16} g_{i-1}^{h}+\frac{10}{16} g_{i}^{h}+\frac{5}{16} g_{i+1}^{h}, \\
g_{2 i+1}^{h+1}=\frac{5}{16} g_{i-1}^{h}+\frac{10}{16} g_{i}^{h}+\frac{1}{16} g_{i+1}^{h}
\end{array}\right.
$$

The symbol of scheme (10) is given by

$$
T(z)=2\left(\frac{z+1}{2}\right)^{5}
$$

Let us now present the class of subdivision schemes, namely, $\mathbf{R}=\left\{R_{\varphi}: \varphi \geq 0, \varphi \in \mathbb{N}\right\}$. The symbol of the scheme $R_{\varphi}$ is obtained by applying symbol of the even part of scheme (8) $\varphi$-times on symbol of scheme (10) and given by

$$
R_{\varphi}(z)=\left(P_{\text {even }}(z)\right)^{\varphi} T(z) .
$$

So by (9), (11), and (12), we have

$$
R_{\varphi}(z)=2\left(\frac{z+1}{2}\right)^{\varphi+5}\left(\frac{-z^{2}+10 z-1}{8}\right)^{\varphi}
$$

where $\{\varphi \geq 0, \varphi \in \mathbb{N}\}$. The members of the class $R$ of subdivision schemes can be categorized by varying $\varphi=0,1,2, \cdots$, in (13). By taking $\varphi=0$ in (13), we get three-point approximating binary scheme [32]. Table 1 presents mask of some members of the proposed class.

\section{Properties of the Proposed Schemes}

In this section, we present some desirable properties of class $\mathbf{R}$ of subdivision schemes, comprising of polynomial generation and reproduction, support, continuity, and Hölder continuity.

4.1. Polynomial Generation and Reproduction. If a subdivision scheme generates polynomials of degree up to $d_{G}$, then the polynomial generation degree of the scheme is $d_{G}$. Also, if the initial data $\mathbf{G}^{0}=\left\{g_{i}^{0}, i \in \mathbb{Z}\right\}$ is sampled from a polynomial $\widehat{\mathbf{P}}$ of degree $d_{R}$ and the scheme yields precisely the same polynomial in the limit, then the reproduction degree $d_{R}$ is the maximal degree of polynomials that can be reproduced by the scheme. Clearly, the reproduction degree is always less than or equal to the generation degree. Now, we establish few results about polynomial generation and polynomial reproduction of the proposed subdivision schemes.

Proposition 5. $R_{\varphi}$-scheme generates space of polynomials up to degree $\varphi+4$.

Proof. Since symbol of $R_{\varphi}$-scheme satisfies the conditions

$R_{\varphi}(1)=2, R_{\varphi}(-1)=0$ and $R_{\varphi}^{j}(-1)=0, j=1,2, \cdots, \varphi+4$,

so by Proposition 3, $R_{\varphi}$-scheme has $\varphi+4$ polynomial generation degree.

In the view of Conti and Hormann [35], the standard parametrization $e_{j}^{h}=j / 2^{h}$ at level $h \in \mathbb{N}$ is not appropriate to analyze a subdivision scheme to reproduce space of polynomials, and the relative shift $\tau_{h}=\left(e_{0}^{h}-e_{0}^{h+1}\right) / 2^{h+1}$ 
TABLE 2: Support, degree of polynomial generation $\left(d_{G}\right)$, degree of polynomial reproduction $\left(d_{R}\right)$, continuity $(C)$, and $\mathrm{HC}$ of $R_{\varphi}$ -scheme for $\varphi=0,1,2$, and 3 .

\begin{tabular}{|c|c|c|c|c|c|c|}
\hline \multirow[t]{2}{*}{$\varphi$} & \multirow[t]{2}{*}{ Support } & \multirow[t]{2}{*}{$d_{G}$} & \multirow[t]{2}{*}{$d_{R}$} & \multirow[t]{2}{*}{ C } & \multicolumn{2}{|c|}{$\begin{array}{c}\text { Hölder } \\
\text { continuity }\end{array}$} \\
\hline & & & & & LB & UB \\
\hline 0 & 5 & 4 & 1 & 3 & 4 & 4 \\
\hline 1 & 8 & 5 & 1 & 4 & 4.678 & 4.678 \\
\hline 2 & 11 & 6 & 1 & 5 & 5.299 & 5.332 \\
\hline 3 & 14 & 7 & 1 & 5 & 5.871 & 5.968 \\
\hline
\end{tabular}

between the parameterizations at iteration level $h$ and $h$ +1 is important for polynomial reproduction of degree $d_{R} \geq 1$. By applying a more suitable parametrization $e_{j}^{h}=$ $\left(j+\tau_{\varphi}\right) / 2^{h}$ with shift parameter $\tau_{\varphi}=R_{\varphi}^{(1)}(1) / 2=(3 \varphi+5) / 2$, we have the following result.

Proposition 6. $R_{\varphi}$-scheme reproduces linear polynomial with respect to parametrization $\left\{e_{j}^{h}=\left(j+\tau_{\varphi}\right) / 2^{h}\right\}_{j \in \mathbb{Z}}$ with shift $\tau_{\varphi}$ $=(3 \varphi+5) / 2$.

Proof. It can be easily verified that

$$
\begin{aligned}
& R_{\varphi}^{(1)}(1)=2 \tau_{\varphi}=3 \varphi+5, \\
& R_{\varphi}^{(j)}(1)=2 \prod_{i=0}^{j-1}\left(\tau_{\varphi}-j\right), \quad j=0,1 .
\end{aligned}
$$

Thus, by Propositions 4 and $5, R_{\varphi}$-scheme reproduces polynomial of degree one.

Table 2 presents the degree of polynomial generation and reproduction of some of the proposed $R_{\varphi}$-schemes. It is observed that the degree of polynomial generation is increasing linearly with the value of parameter $\varphi$.

4.2. Support. The support of a subdivision scheme quantifies how much one vertex brought change in its neighboring vertices, and its measure represents local support of the limit curve. Basic limit function (BLF) of a convergent subdivision scheme is a limit function of the initial data $\mathbf{G}^{0}=\left\{g_{i}^{0}, i \in \mathbb{Z}\right\}$ which is of the form

$$
g_{i}^{0}= \begin{cases}1, & i=0 \\ 0, & i \neq 0\end{cases}
$$

By following [36], we determine that support of BLF of $R_{\varphi}$-scheme is $3 \varphi+5$. BLF generated by the proposed $R_{0}$ and $R_{1}$ schemes are demonstrated in Figure 1.

4.3. Continuity Analysis. Continuity of a subdivision scheme is an essential parameter on which efficiency of a scheme depends. To investigate continuity of our proposed class, we follow the approach as given in [33] and use the symbol of $R_{\varphi}$-scheme.

Theorem 7. The $R_{\varphi}$-scheme has $C^{\varphi+3-v_{\varphi}}$ continuity, where $\varphi$ $=1,2,3, \cdots$, and $v_{\varphi}=\lfloor(\varphi-1) / 2\rfloor$ (floor function).

Proof. The symbol of $R_{\varphi}$-scheme (13) can be simplified as

$$
R_{\varphi}(z)=\frac{(z+1)^{\varphi+4-v_{\varphi}}}{2^{\varphi+3-v_{\varphi}}} r_{\varphi}(z)
$$

with

$$
r_{\varphi}(z)=\left(\frac{z+1}{2}\right)^{1+v_{\varphi}}\left(\frac{-z^{2}+10 z-1}{8}\right)^{\varphi}, \quad \varphi=1,2,3, \cdots,
$$

where $v_{\varphi}=\lfloor(\varphi-1) / 2\rfloor$.

Let $S_{r_{\varphi}}$ be the subdivision scheme associated with the symbol $r_{\varphi}(z)$. The scheme $S_{r_{\varphi}}$ is contractive provided that $\left\|S_{r_{\varphi}}\right\|_{\infty}=\max \left\{\sum_{l \in \mathbb{Z}}\left|r_{2 l}\right|, \sum_{l \in \mathbb{Z}}\left|r_{2 l+1}\right|\right\}<1$. So, by Theorem 2, $R_{\varphi}$-scheme has $C^{\varphi+3-V_{\varphi}}$ continuity.

In Theorem 7, we discuss continuity of $R_{\varphi}$-scheme for $\varphi=1,2,3, \cdots$. It is to be noted that $R_{0}$-scheme has $C^{3}$ continuity which is analyzed in [32].

Corollary 8. The $R_{1}$-scheme has $C^{4}$ continuity.

Proof. By letting $\varphi=1$, the symbol of $R_{1}$-scheme from (17) and (18) is given by

$$
R_{1}(z)=\frac{(z+1)^{5}}{2^{4}} r_{1}(z)
$$

with

$$
r_{1}(z)=\frac{1}{16}\left(-z^{3}+9 z^{2}+9 z-1\right)
$$

Let $S_{r_{1}}$ be the scheme corresponding to the symbol $r_{1}(z)$. The scheme $S_{r_{1}}$ is contractive, as $\left\|S_{r_{1}}\right\|_{\infty}=\max \{10 / 16,10 /$ $16\}=(10 / 16)<1$. So, by Theorem $7, R_{1}$-scheme has $C^{4}$ continuity.

Similarly, for different values of parameter $\varphi$, continuity of $R_{\varphi}$-scheme can be easily computed by using Theorem 7 .

4.4. Hölder Continuity Analysis. Continuity of a subdivision scheme is related to the existence of derivative of subdivision curve. For example, subdivision curve is said to be $C^{m}$ continuous if the $m^{\text {th }}$ derivative of the curve exists and is continuous everywhere in the given interval. On the other hand, 


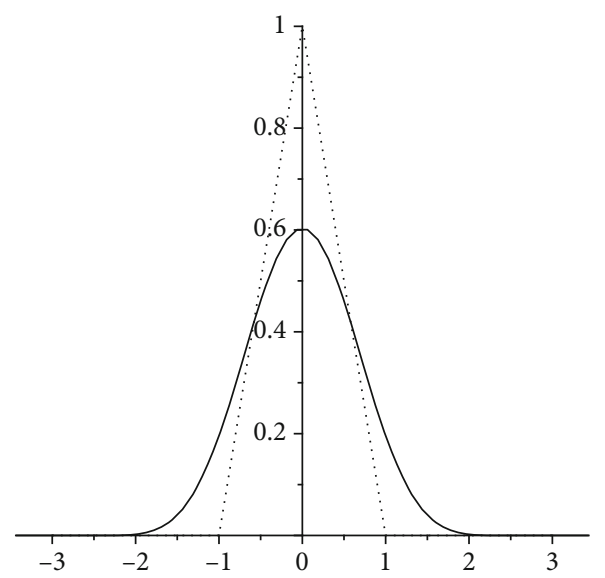

(a)

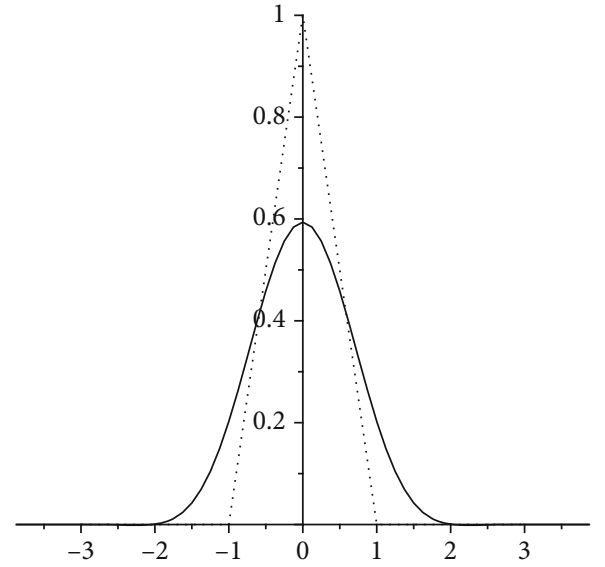

(b)

Figure 1: (a, b) Basic limit functions generated by the proposed schemes $R_{0}$ and $R_{1}$, respectively.

Hölder continuity (HC) of a subdivision scheme tells how continuous the highest continuous derivative is. Therefore, it is also important to find $\mathrm{HC}$ of subdivision schemes along with continuity. Lower bound (LB) on HC of the proposed class is calculated by using an interesting property of symbol of $R_{\varphi}$-scheme, i.e., odd coefficients in $R_{\varphi}(z)$ are nonnegative and even coefficients are nonpositive.

Theorem 9. $L B$ on the $H C$ of $R_{\varphi}$-scheme is $\varphi+5-\log _{2}$ $\left((3 / 2)^{\varphi}+1\right)$, where $\varphi=0,1,2, \cdots$.

Proof. By (13), symbol of $R_{\varphi}$-scheme can be expressed as

$$
R_{\varphi}(z)=\left(\frac{z+1}{2}\right)^{\varphi+5} U_{\varphi}(z)
$$

where $U_{\varphi}(z)=(a(z))^{\varphi} b(z), a(z)=\left(-z^{2}+10 z-1\right) / 8$, and $b$ $(z)=2$. So LB on HC of $R_{\varphi}$-scheme is given by $\varphi+5-$ $\log _{2}\left\|U_{\varphi}\right\|$. As we know $\left\|U_{\varphi}\right\|=\max \left(u^{\diamond}, u_{\diamond}\right)$, where $u^{\diamond}$ is the sum of odd and $u_{\diamond}$ is the sum of even coefficients of $U_{\varphi}(z)$. We can write coefficients of $U_{\varphi}(z)$ in the following manner:

$$
\left(\begin{array}{l}
u^{\diamond} \\
u^{\diamond}
\end{array}\right)=\left(\begin{array}{ll}
a^{\diamond} & a_{\diamond} \\
a_{\diamond} & a^{\diamond}
\end{array}\right)^{\varphi}\left(\begin{array}{l}
b^{\diamond} \\
b^{\diamond}
\end{array}\right) \text {. }
$$

Thus, we have

$$
\left(\begin{array}{l}
u^{\diamond} \\
u^{\diamond}
\end{array}\right)=\left(\begin{array}{cc}
5 & -\frac{1}{4} \\
\frac{1}{4} & \frac{5}{4}
\end{array}\right)^{\varphi}\left(\begin{array}{l}
0 \\
2
\end{array}\right) \text {. }
$$

By eigenvalue decomposition, we have

$$
\left(\begin{array}{l}
u^{\circ} \\
u^{\circ}
\end{array}\right)=\frac{1}{2}\left(\begin{array}{cc}
-1 & 1 \\
1 & 1
\end{array}\right)\left(\begin{array}{cc}
3 & 0 \\
0 & 1
\end{array}\right)^{\varphi}\left(\begin{array}{cc}
-1 & 1 \\
1 & 1
\end{array}\right)\left(\begin{array}{l}
0 \\
2
\end{array}\right) \text {, }
$$

which implies that

$$
\left(\begin{array}{l}
u^{\diamond} \\
u^{\diamond}
\end{array}\right)=\left(\begin{array}{cc}
-\left(\frac{3}{2}\right)^{\varphi} & +1 \\
\left(\frac{3}{2}\right)^{\varphi} & +1
\end{array}\right)^{\varphi} .
$$

Thus, we have

$$
\left\|U_{\varphi}\right\|=\left(\frac{3}{2}\right)^{\varphi}+1
$$

Consequently, LB on $\mathrm{HC}$ of $R_{\varphi}$-scheme is $\varphi+5-\log _{2}$ $\left((3 / 2)^{\varphi}+1\right)$, where $\varphi=0,1, \cdots$.

Upper bound (UB) on $\mathrm{HC}$ of $R_{\varphi}$-scheme is as follows.

Theorem 10. UB on HC of $R_{\varphi}$-scheme is $\varphi+5-\log _{2}\left(\zeta_{\varphi}\right)$, where $\varphi=0,1, \cdots$, and $\zeta_{\varphi}$ be the joint spectral radius of the matrices $Q_{0}$ and $Q_{1}$ which are obtained by using symbol of $R_{\varphi}$-scheme.

Proof. By (13), symbol of $R_{\varphi}$-scheme can be expressed as

$$
R_{\varphi}(z)=\left(\frac{z+1}{2}\right)^{\varphi+5} Q_{\varphi}(z),
$$

where $Q_{\varphi}(z)=2\left(\left(-z^{2}+10 z-1\right) / 8\right)^{\varphi}$. Let $q_{0}, q_{1}, \cdots, q_{d}$ be the nonzero real coefficients of $Q_{\varphi}(z)$. Also, $Q_{0}$ and $Q_{1}$ are the matrices of order $d \times d$ defined by 


$$
\left(Q_{0}\right)_{m n}=q_{d+m-2 n} \text {, and }\left(Q_{1}\right)_{m n}=q_{d+m-2 n+1},
$$

where $m, n=1,2, \cdots, d$.

Let us denote joint spectral radius of both matrices $Q_{0}$ and $Q_{1}$ by $\zeta_{\varphi}$. Then, by Rioul [37] and Dyn [33], UB on $\mathrm{HC}$ of $R_{\varphi}$-scheme is given by $\varphi+5-\log _{2}\left(\zeta_{\varphi}\right)$.

For different values of parameter $\varphi$, upper and lower bounds on the $\mathrm{HC}$ of $R_{\varphi}$-schemes can be straightforwardly computed by using Theorems 9 and 10. Table 2 summarizes the continuity and $\mathrm{HC}$ of the proposed class of subdivision schemes. It clearly indicates that as we go up for higher values of parameter $\varphi$, continuity and $\mathrm{HC}$ of $R_{\varphi}$-schemes also increase. Moreover, newly generated $R_{\varphi}$-schemes have higher order of continuity and HC as compared to their parent subdivision schemes.

\section{Geometrical Analysis of Proposed Schemes}

The shape of an object is generally controlled by a control polygon. The purpose of applying a subdivision scheme on the control polygon is to generate visually smooth curves. Figure 2 presents the behavior of some of the proposed schemes. $R_{0}, R_{1}$, and $R_{2}$ schemes are applied on the same initial polygon, and limit curves are obtained after three iterations. It is evident that the proposed class offers more choices to meet different designing needs.

5.1. Subdivision Rules for Endpoints. For closed curves, the subdivision rules of $R_{0}, R_{1}, R_{2}$, and $R_{3}$ schemes can be defined by their corresponding Laurent polynomial from (13). The limit curves generated by these schemes are $C^{3}, C^{4}, C^{5}$, and $C^{5}$ continuous, respectively. In case of dealing with open polygons, these rules can be used to improve the interior of the curve, while it is quite troublesome to improve the first and last edges with the help of subdivision rules of the original proposed schemes. So to handle the endpoints of an open polygon, we need to supply additional points which are not usually required in case of a closed polygon. Let $g_{0}^{h} g_{1}^{h}$ be the first edge of the open polygon $\left\{G^{h}=g_{k}^{h}: k=0, \cdots, 2^{h} m\right\}$. Now, we define an additional control point $g_{-1}^{h}$, as an extrapolatory rule in the nonrefined polygon $G^{h}$, and then we can compute the point $g_{1}^{h+1}$ through the proposed schemes by applying subdivision to the subpolygon $\left\{g_{-1}^{h}, g_{0}^{h}, g_{1}^{h}, g_{2}^{h}\right\}$. We select the point as $g_{-1}^{h}=2 g_{0}^{h}-g_{1}^{h}$. The first edge of an open control polygon $\left\{G^{h}=g_{k}^{h}: k=0,1, \cdots, 2^{h} m\right\}$ can be refined by using the following rules.

(i) Refinement rules of the proposed three-point scheme $R_{0}$ are given by

$$
\begin{aligned}
& g_{0}^{h+1}=\frac{5}{4} g_{0}^{h}-\frac{1}{4} g_{1}^{h}, \\
& g_{1}^{h+1}=\frac{3}{4} g_{0}^{h}+\frac{1}{4} g_{1}^{h} .
\end{aligned}
$$

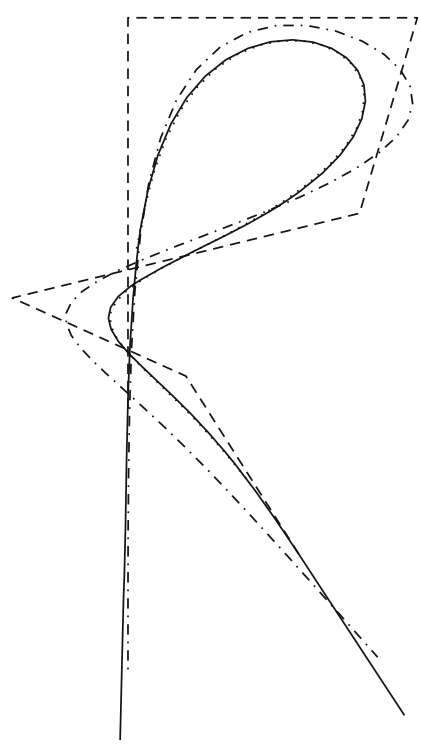

$$
\begin{aligned}
& \text { - - Original } \\
& \text {-. - The scheme } R_{0}\left(C^{3}\right) \\
& \text { - The scheme } R_{1}\left(C^{4}\right) \\
& \ldots \ldots \text { The scheme } R_{2}\left(C^{5}\right)
\end{aligned}
$$

FIgURE 2: Behavior of the proposed $R_{0}, R_{1}$, and $R_{2}$ schemes after three iterations.

(ii) Refinement rules of the proposed five-point scheme $R_{1}$ are given by

$$
\begin{aligned}
& g_{0}^{h+1}=\frac{380}{256} g_{0}^{h}-\frac{120}{256} g_{1}^{h}-\frac{4}{256} g_{2}^{h}, \\
& g_{1}^{h+1}=\frac{255}{256} g_{0}^{h}+\frac{2}{256} g_{1}^{h}-\frac{1}{256} g_{2}^{h}, \\
& g_{2}^{h+1}=\frac{132}{256} g_{0}^{h}+\frac{120}{256} g_{1}^{h}+\frac{4}{256} g_{2}^{h}, \\
& g_{3}^{h+1}=\frac{42}{256} g_{0}^{h}+\frac{171}{256} g_{1}^{h}+\frac{44}{256} g_{2}^{h}-\frac{1}{256} g_{3}^{h} .
\end{aligned}
$$

(iii) Refinement rules of the proposed six-point scheme $R_{2}$ are given by

$$
\begin{aligned}
& g_{0}^{h+1}=\frac{3070}{4096} g_{0}^{h}+\frac{1029}{4096} g_{1}^{h}-\frac{4}{4096} g_{2}^{h}+\frac{1}{4096} g_{3}^{h}, \\
& g_{1}^{h+1}=\frac{1306}{4096} g_{0}^{h}+\frac{2495}{4096} g_{1}^{h}+\frac{308}{4096} g_{2}^{h}-\frac{13}{4096} g_{3}^{h}, \\
& g_{2}^{h+1}=\frac{283}{4096} g_{0}^{h}+\frac{2491}{4096} g_{1}^{h}+\frac{1338}{4096} g_{2}^{h}-\frac{17}{4096} g_{3}^{h}+\frac{1}{4096} g_{4}^{h}, \\
& g_{3}^{h+1}=-\frac{15}{4096} g_{0}^{h}+\frac{1337}{4096} g_{1}^{h}+\frac{2478}{4096} g_{2}^{h}+\frac{309}{4096} g_{3}^{h}-\frac{13}{4096} g_{4}^{h}
\end{aligned}
$$




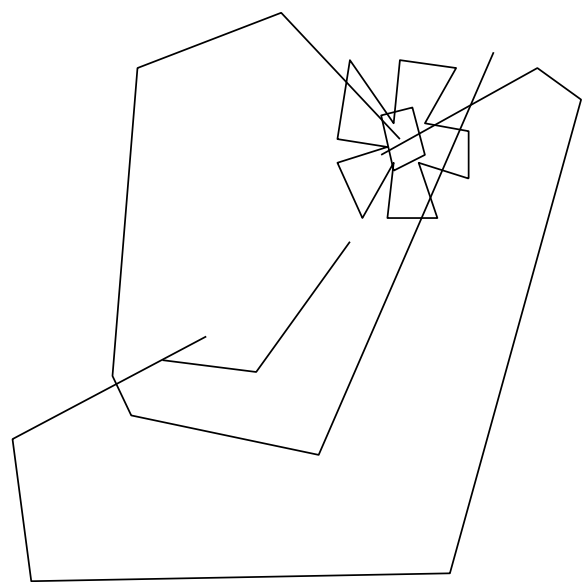

(a)

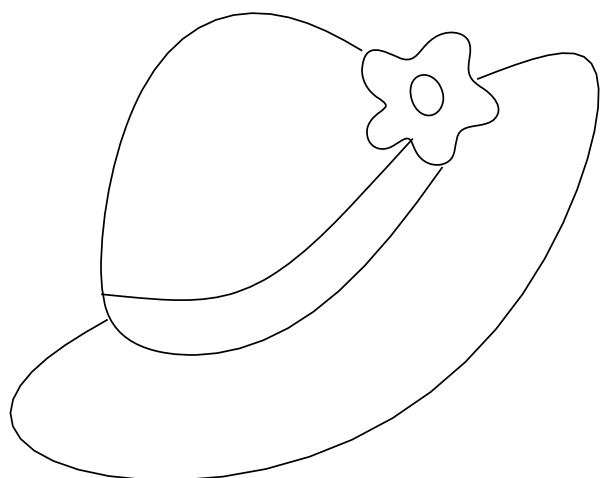

(b)

Figure 3: Application of $R_{0}$-scheme: (a) initial polygon and (b) the limit curve generated by $R_{0}$-scheme at the third subdivision level.

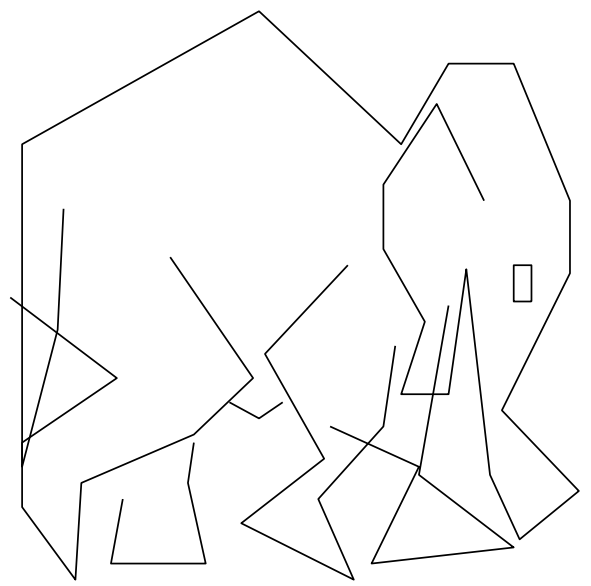

(a)

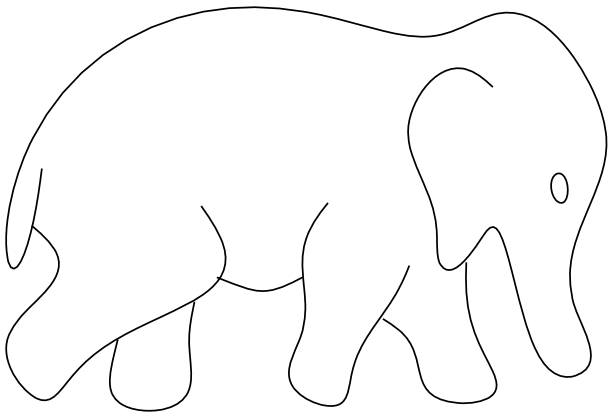

(b)

FIGURE 4: Application of $R_{0}$-scheme: (a) initial polygon and (b) the limit curve generated by $R_{0}$-scheme at the third subdivision level.

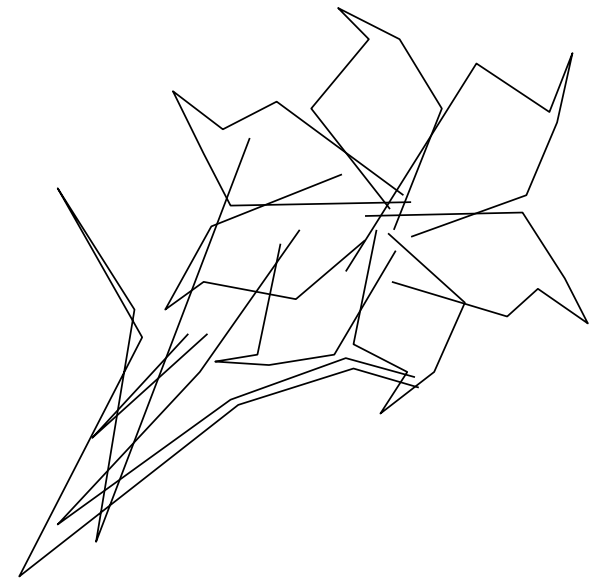

(a)

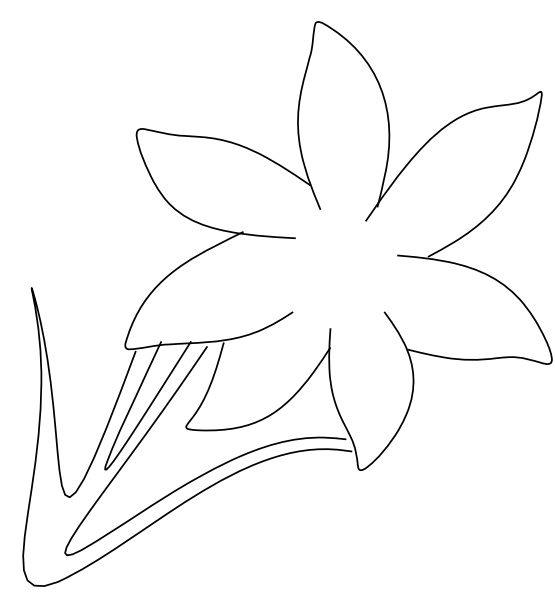

(b)

FIgURE 5: Application of $R_{1}$-scheme: (a) initial polygon and (b) the limit curve generated by $R_{1}$-scheme at the third subdivision level. 


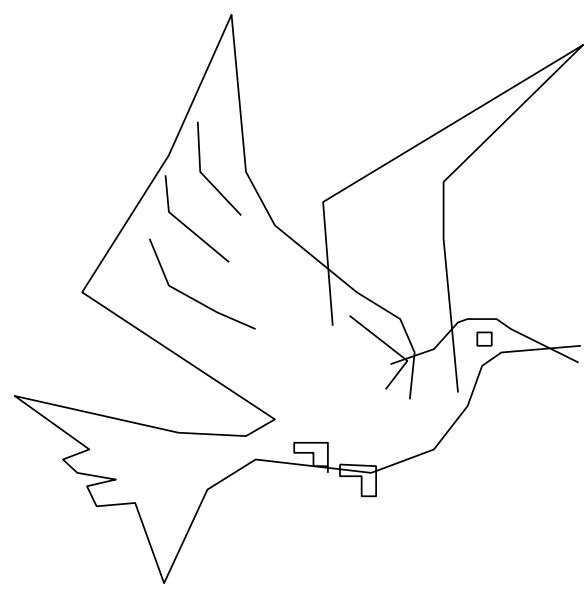

(a)

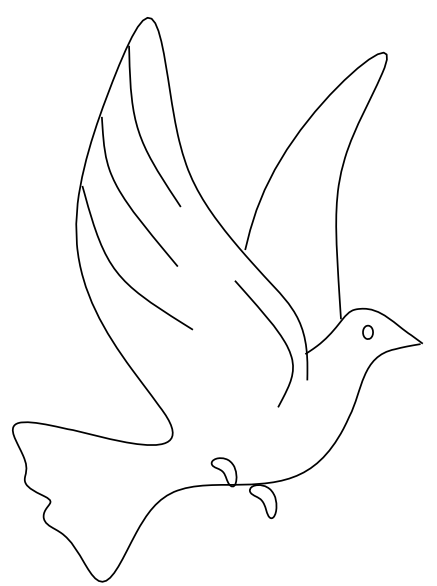

(b)

Figure 6: Application of $R_{1}$-scheme: (a) initial polygon and (b) the limit curve generated by $R_{1}$-scheme at the third subdivision level.

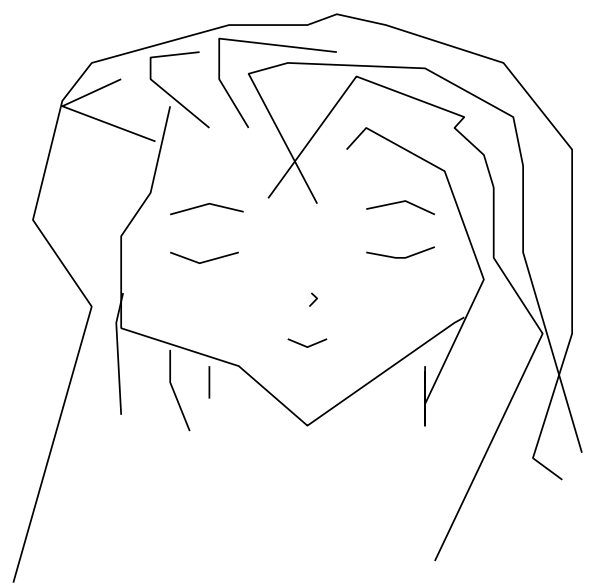

(a)

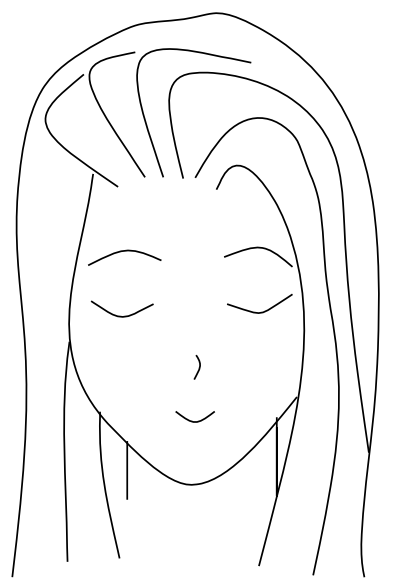

(b)

FIgURE 7: Application of $R_{2}$-scheme: (a) initial polygon and (b) the limit curve generated by $R_{2}$-scheme at the third subdivision level.

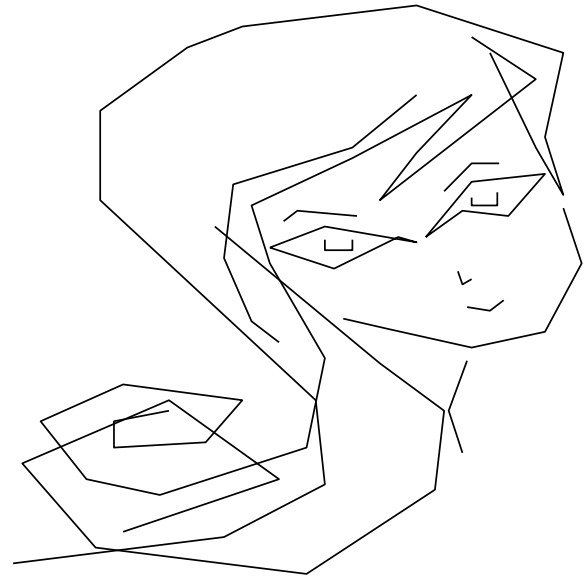

(a)

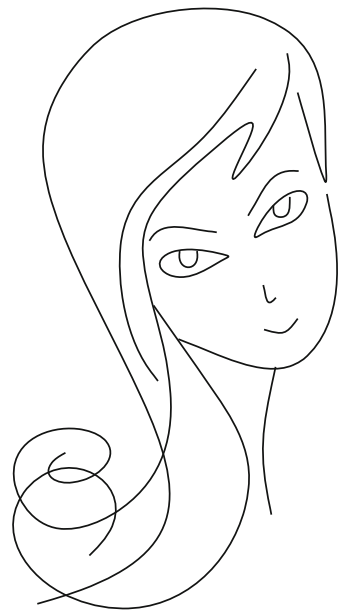

(b)

Figure 8: Application of $R_{3}$-scheme: (a) initial polygon and (b) the limit curve generated by $R_{3}$-scheme at the third subdivision level. 


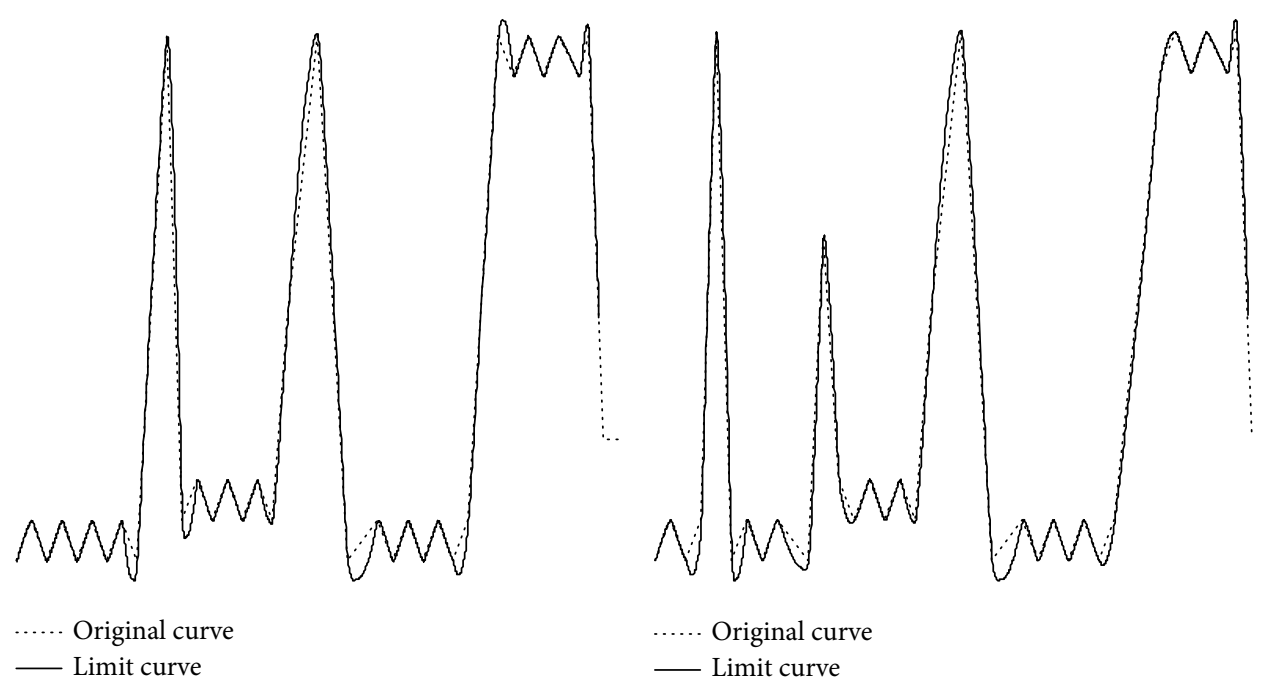

(a)

(b)

Figure 9: Application of $R_{3}$-scheme: $(\mathrm{a}, \mathrm{b})$ the initial polygon along with sharp features of limit curve generated by $R_{3}$-scheme at the third subdivision level.

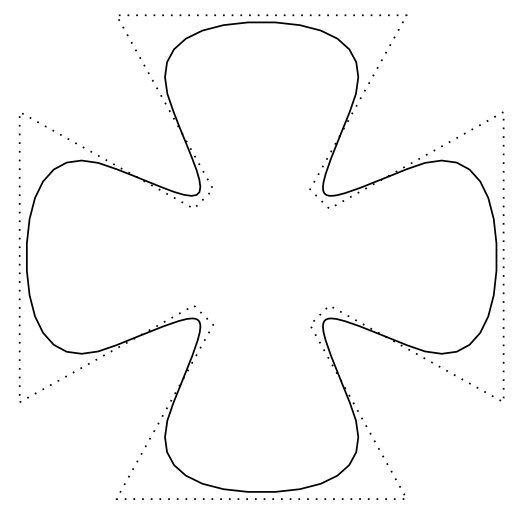

(a) 3-point scheme [38]

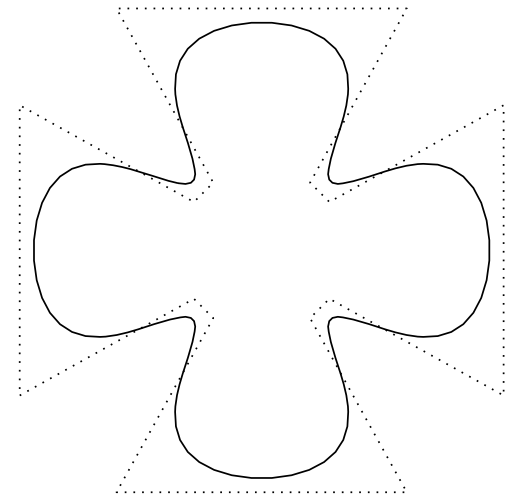

(d) $R_{0}$-scheme

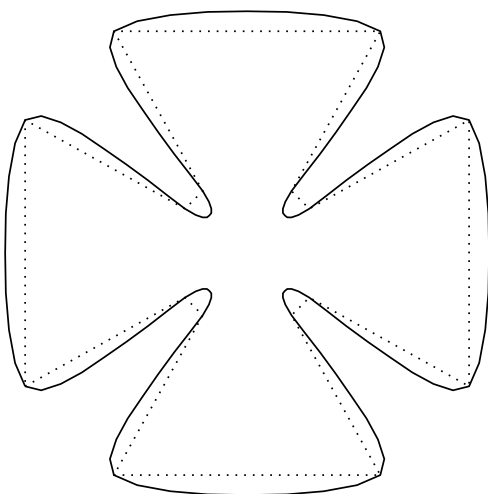

(b) 4-point scheme [18]

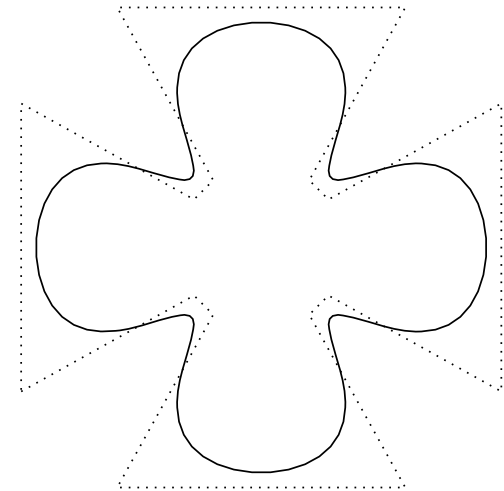

(e) $R_{1}$-scheme

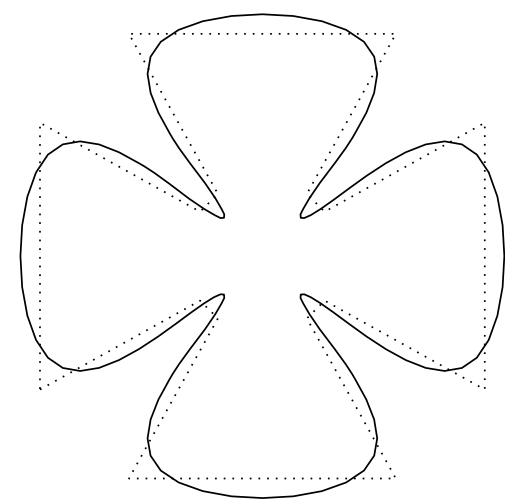

(c) 5-point scheme [19]

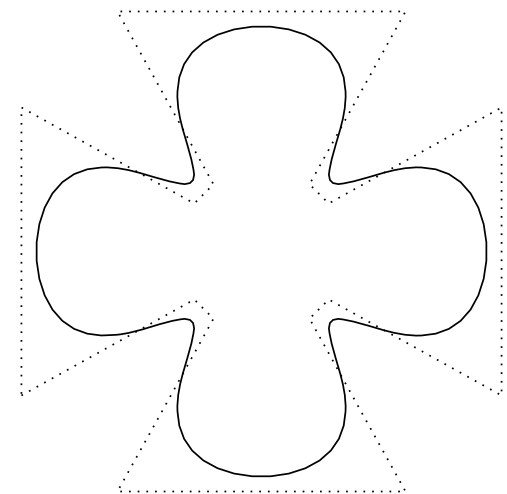

(f) $R_{2}$-scheme

FIGURE 10: Comparison of the existing and proposed subdivision schemes at the third subdivision level. 


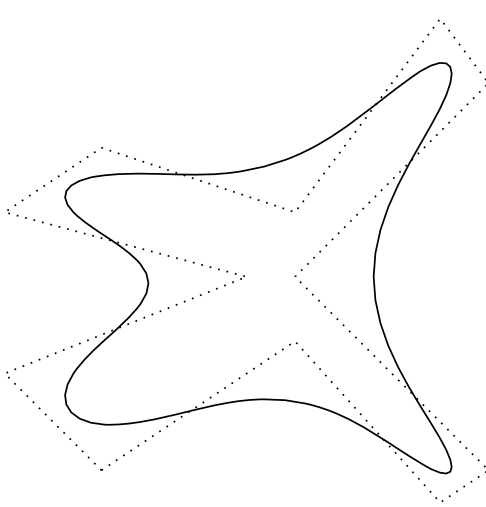

(a) 3-point scheme [38]

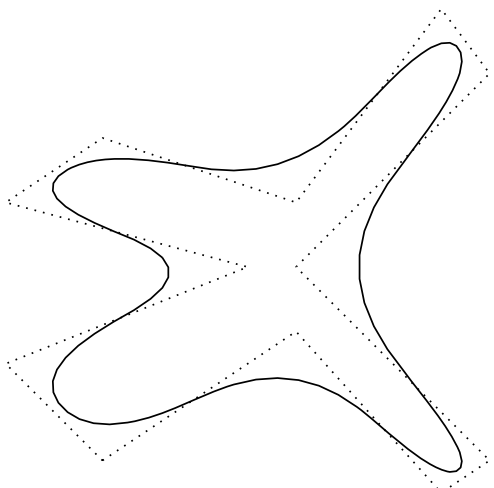

(d) $R_{0}$-scheme

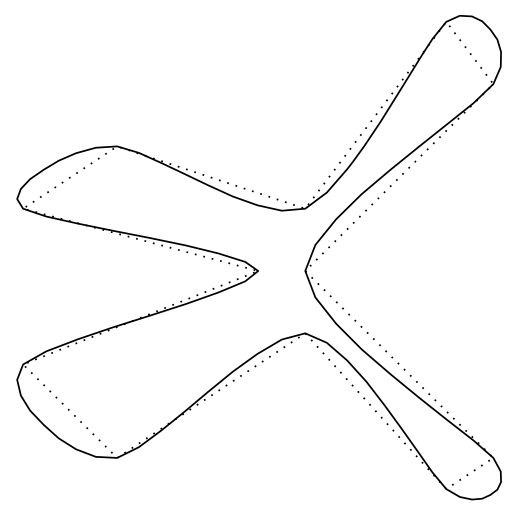

(b) 4-point scheme [18]

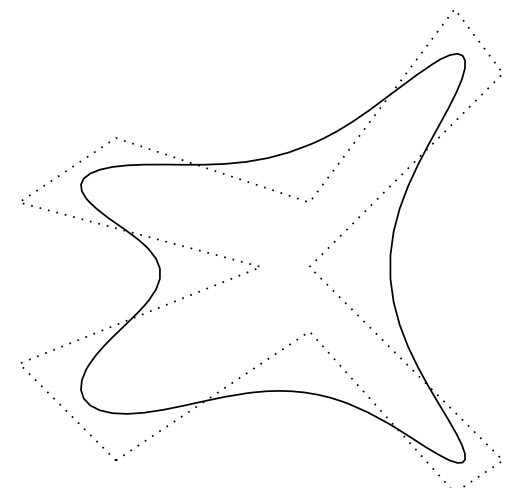

(e) $R_{1}$-scheme

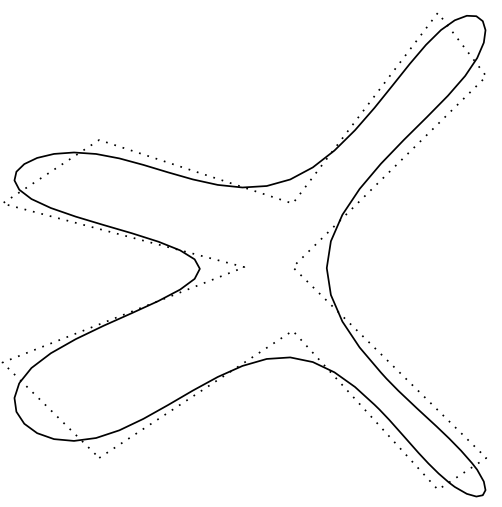

(c) 5-point scheme [19]

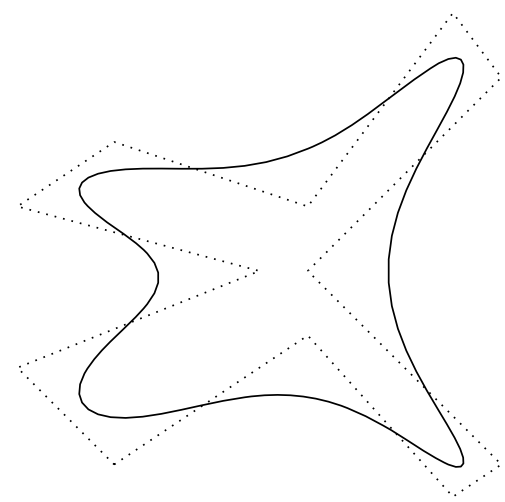

(f) $R_{2}$-scheme

Figure 11: Comparison of the existing and proposed subdivision schemes at the third subdivision level.

(iv) Refinement rules of the proposed eight-point scheme $R_{3}$ are given by

$$
\begin{aligned}
g_{0}^{h+1}= & \frac{65066}{65536} g_{0}^{h}+\frac{1028}{65536} g_{1}^{h}-\frac{580}{65536} g_{2}^{h}+\frac{22}{65536} g_{3}^{h}, \\
g_{1}^{h+1}= & \frac{33976}{65536} g_{0}^{h}+\frac{30254}{65536} g_{1}^{h}+\frac{1394}{65536} g_{2}^{h}-\frac{90}{65536} g_{3}^{h} \\
& -\frac{1}{65536} g_{4}^{h}, \\
g_{2}^{h+1}= & \frac{11246}{65536} g_{0}^{h}-\frac{42508}{65536} g_{1}^{h}+\frac{12340}{65536} g_{2}^{h}-\frac{580}{65536} g_{3}^{h} \\
& +\frac{22}{65536} g_{4}^{h}, \\
g_{3}^{h+1}= & \frac{1119}{65536} g_{0}^{h}+\frac{31648}{65536} g_{1}^{h}+\frac{31558}{65536} g_{2}^{h}+\frac{1303}{65536} g_{3}^{h} \\
& -\frac{91}{65536} g_{4}^{h}-\frac{1}{65536} g_{5}^{h}, \\
g_{4}^{h+1}= & \frac{536}{65536} g_{0}^{h}-\frac{12340}{65536} g_{1}^{h}+\frac{41928}{65536} g_{2}^{h}+\frac{12362}{65536} g_{3}^{h} \\
& -\frac{580}{65536} g_{4}^{h}+\frac{22}{65536} g_{5}^{h}, \\
g_{5}^{h+1}= & -\frac{93}{65536} g_{0}^{h}+\frac{1304}{65536} g_{1}^{h}+\frac{31557}{65536} g_{2}^{h}+\frac{31557}{65536} g_{3}^{h} \\
& +\frac{1303}{65536} g_{4}^{h}-\frac{91}{65536} g_{5}^{h}-\frac{1}{65536} g_{6}^{h} .
\end{aligned}
$$

Similarly, we can refine the final edges of the open polygon.
5.2. Applications and Comparison. Geometrical performance of $R_{0}, R_{1}, R_{2}$, and $R_{3}$ schemes is depicted through several examples. The proposed schemes have good continuity and present smooth limit curves. Figures 3(a) and 4(a) present initial control polygons of cap and elephant, respectively, while Figures 3(b) and 4(b) are the limit curves obtained by applying three iterations of $R_{0}$-scheme on these initial polygons. Figures 5(a) and 6(a) present initial control polygons of flower and bird, respectively, while Figures 5(b) and 6(b) are the limit curves obtained by applying three iterations of $R_{1}$-scheme on these initial polygons. Figures $7(\mathrm{a})$ and $8(\mathrm{a})$ present initial control polygons of face of girls, while Figures 7(b) and 8(b) are the limit curves obtained by applying three iterations of $R_{2}$-scheme on these initial polygons, respectively.

Figure 9 represents the initial polygon along with sharp features of limit curve generated by $R_{3}$-scheme at the third subdivision level. Figures 10 and 11 present comparison of some existing subdivision schemes (3-point scheme [38], 4point scheme [18], and 5-point scheme [19]) with the proposed subdivision schemes $\left(R_{0}, R_{1}\right.$, and $R_{2}$ schemes). We have chosen two different initial polygons, and limit curves are generated after three subdivision levels. It is clear from the figures that the proposed schemes generate smooth limit curves.

\section{Conclusion}

Subdivision is an efficient way of constructing smooth curves or surfaces in geometric modeling and computer graphics. In 
this paper, we have presented an elegant way of constructing a class of approximating binary subdivision schemes by using two well-known binary subdivision schemes. Several examples are provided to illustrate that the proposed schemes give wide choice to geometric designers for generation of smooth geometric models as per their own needs. Comparison with some existing schemes is also given. Moreover, several important properties like polynomial reproduction and generation, support of BLF, continuity, and $\mathrm{HC}$ of the proposed scheme are discussed. Geometrical analysis of the limit curve is also carried out.

\section{Data Availability}

No data were used in this study.

\section{Conflicts of Interest}

The authors declare that they have no competing interests.

\section{Authors' Contributions}

All the authors contributed equally, and they read and approved the final manuscript for publication.

\section{Acknowledgments}

Taif University Researchers Supporting Project number (TURSP-2020/20), Taif University, Taif, Saudi Arabia.

\section{References}

[1] M. Shakeel, I. Hussain, H. Ahmad, I. Ahmad, P. Thounthong, and Y. F. Zhang, "Meshless technique for the solution of timefractional partial differential equations having real-world applications," Journal of Function Spaces, vol. 2020, Article ID 8898309, 17 pages, 2020.

[2] S. Khaldi, R. Mecheraoui, and A. Mukheimer, "A nonlinear fractional problem with mixed Volterra-Fredholm integrodifferential equation: existence, uniqueness, H-U-R stability, and regularity of solutions," Journal of Function Spaces, vol. 2020, Article ID 4237680, 14 pages, 2020.

[3] G. Mustafa, S. T. Ejaz, D. Baleanu, A. Ghaffar, and K. S. Nisar, "A subdivision-based approach for singularly perturbed boundary value problem," Advances in Difference Equations, vol. 1, 20 pages, 2020.

[4] M. K. Jena, P. Shunmugaraj, and P. C. Das, "A subdivision algorithm for trigonometric spline curves," Computer Aided Geometric Design, vol. 19, no. 1, pp. 71-88, 2002.

[5] B.-j. Li, Z.-l. Yu, B.-w. Yu, S. U. Zhi-xun, and X.-p. Liu, "Nonstationary subdivision for exponential polynomials reproduction," Acta Mathematicae Applicatae Sinica, English Series, vol. 29, no. 3, pp. 567-578, 2013.

[6] G. Mustafa, F. Khan, and A. Ghaffar, "The m-point approximating subdivision scheme," Lobachevskii Journal of Mathematics, vol. 30, no. 2, pp. 138-145, 2009.

[7] G. Mustafa, A. Ghaffar, and F. Khan, "The odd-point ternary approximating schemes," American Journal of Computational Mathematics, vol. 1, no. 2, pp. 111-118, 2011.
[8] W. Salam, S. S. Siddiqi, and K. Rehan, "Chaikin's perturbation subdivision scheme in non-stationary forms," Alexandria Engineering Journal, vol. 55, no. 3, pp. 2855-2862, 2016.

[9] S. S. Siddiqi and M. Younis, "Ternary approximating nonstationary subdivision schemes for curve design," Central European Journal of Engineering, vol. 4, pp. 371-378, 2014.

[10] S. S. Siddiqi and N. Ahmad, “An approximating $C^{4}$ stationary subdivision scheme," European Journal of Scientific Research, vol. 15, pp. 97-102, 2006.

[11] A. Shahzad, F. Khan, A. Ghaffar, G. Mustafa, K. S. Nisar, and D. Baleanu, "A novel numerical algorithm to estimate the subdivision depth of binary subdivision schemes," Symmetry, vol. 12, no. 1, p. 66, 2020.

[12] S. M. Hussain, A. U. Rehman, D. Baleanu, K. S. Nisar, A. Ghaffar, and S. A. Abdul Karim, "Generalized 5-point approximating subdivision scheme of varying arity," Mathematics, vol. 8, no. 4, p. 474, 2020.

[13] X. Song, M. Aigner, F. Chen, and B. Jüttler, "Circular spline fitting using an evolution process," Journal of Computational and Applied Mathematics, vol. 231, no. 1, pp. 423-433, 2009.

[14] J. Tan, J. Sun, and G. Tong, "A non-stationary binary threepoint approximating subdivision scheme," Applied Mathematics and Computation, vol. 276, pp. 37-43, 2016.

[15] J. Wallner and N. Dyn, "Convergence and $C^{1}$ analysis of subdivision schemes on manifolds by proximity," Computer Aided Geometric Design, vol. 22, pp. 593-622, 2005.

[16] L. Zhang, H. Ma, S. Tang, and J. Tan, "A combined approximating and interpolating ternary 4-point subdivision scheme," Journal of Computational and Applied Mathematics, vol. 350, pp. 37-49, 2018.

[17] P. Ashraf, A. Ghaffar, D. Baleanu, I. Sehar, K. S. Nisar, and F. Khan, "Shape-preserving properties of a relaxed four-point interpolating subdivision scheme," Mathematics, vol. 8, no. 5, p. 806, 2020.

[18] G. Deslauriers and S. Dubuc, "Symmetric iterative interpolation processe," Constructive Approximation, vol. 5, no. 5, pp. 49-68, 1989.

[19] K. Hormann and M. A. Sabin, "A family of subdivision schemes with cubic precision," Computer Aided Geometric Design, vol. 25, no. 1, pp. 41-52, 2008.

[20] G. Mustafa, P. Ashraf, and M. Aslam, "Binary univariate dual and primal subdivision schemes," SeMA Journal, vol. 65, no. 1, pp. 23-35, 2014.

[21] P. Ashraf, G. Mustafa, and J. Deng, "A six-point variant on the Lane-Riesenfeld algorithm," Journal of Applied Mathematics, vol. 2014, Article ID 628285, 7 pages, 2014.

[22] G. Mustafa and M. Bari, "Wide-ranging families of subdivision schemes for fitting data," Punjab University Journal of Mathematics, vol. 48, no. 2, pp. 125-134, 2016.

[23] M. Asghar and G. Mustafa, "Stationary and non-stationary univariate subdivision schemes," Punjab University Journal of Mathematics, vol. 50, no. 3, pp. 25-42, 2018.

[24] A. Ghaffar, G. Mustafa, and K. Qin, "Unification and application of 3-point approximating subdivision schemes of varying arity," Open Journal of Applied Sciences, vol. 2, no. 4, pp. 4852, 2012.

[25] A. Ghaffar, G. Mustafa, and K. Qin, "The 4-point $\alpha$-ary approximating subdivision scheme," Open Journal of Applied Sciences, vol. 3, no. 1, pp. 106-111, 2013.

[26] A. Ghaffar, Z. Ullah, M. Bari, K. S. Nisar, M. M. Al-Qurashi, and D. Baleanu, "A new class of $2 m$-point binary non- 
stationary subdivision schemes," Advances in Difference Equations, vol. 2019, 2019.

[27] A. Ghaffar, Z. Ullah, M. Bari, K. S. Nisar, and D. Baleanu, "Family of odd point non-stationary subdivision schemes and their applications," Advances in Difference Equations, vol. 2019, no. 1, p. 20, 2019.

[28] A. Ghaffar, M. Bari, Z. Ullah, M. Iqbal, K. S. Nisar, and D. Baleanu, "A new class of 2q-point nonstationary subdivision schemes and their applications," Mathematics, vol. 7, no. 7, p. 639, 2019.

[29] A. Ghaffar, M. Iqbal, M. Bari et al., "Construction and application of nine-tic B-spline tensor product SS," Mathematics, vol. 7, no. 8, p. 675, 2019.

[30] P. Ashraf, B. Nawaz, D. Baleanu et al., "Analysis of geometric properties of ternary four-point rational interpolating subdivision scheme," Mathematics, vol. 8, no. 3, p. 338, 2020.

[31] P. Ashraf, M. Sabir, A. Ghaffar, K. S. Nisar, and I. Khan, "Shape-preservation of ternary four-point interpolating nonstationary subdivision scheme," Frontiers of Physics, vol. 7, 2020.

[32] M. F. Hassan and N. A. Dodgson, "Ternary three-point univariate subdivision schemes," in Curve and Surface Fitting: Saint-Malo (2002), pp. 199-208, Nashboro Press, Brentwood, 2003.

[33] N. Dyn and D. Levin, "Subdivision schemes in geometric modelling," Acta Numerica, vol. 11, pp. 73-144, 2002.

[34] N. Dyn, K. Hormann, M. A. Sabin, and Z. Shen, "Polynomial reproduction by symmetric subdivision schemes," Journal of Approximation Theory, vol. 155, no. 1, pp. 28-42, 2008.

[35] C. Conti and K. Hormann, "Polynomial reproduction for univariate subdivision schemes of any arity," Journal of Approximation Theory, vol. 163, no. 4, pp. 413-437, 2011.

[36] I. P. Ivrissimtzis, M. A. Sabin, and N. A. Dodgson, "On the support of recursive subdivision," ACM Transactions on Graphics, vol. 23, no. 4, pp. 1043-1060, 2004.

[37] O. Rioul, "Simple regularity criteria for subdivision schemes," SIAM Journal on Mathematical Analysis, vol. 23, no. 6, pp. 1544-1576, 1992.

[38] G. Mustafa, P. Ashraf, and N. Saba, "A new class of binary approximating subdivision schemes," Jurnal Teknologi (Sciences and Engineering), vol. 78, no. 4, pp. 65-72, 2016. 